\title{
Mémoire, histoire et identités sociales: Le role des représentations sociales dans la formation des identités sociales et dans la construction de la connaissance historique par les élèves
}

\author{
Lana Mara de Castro Siman \\ Universidade Federal de Minas Gerais- Brasil

\section{RÉSUMÉ}

Dans cet article, nous discuterons comment des représentations de la population noire, socialement construites dans le passé et véhiculées par la mémoire persistent dans l'imaginaire des élèves et requièrent, de la part des professeurs d'histoire, une attention toute particulière quant à leur déconstruction et reconstruction. Cette discussion sera basée sur des données recueillies dans des cours d'histoire de classes d'enfants de 9 à Ir ans et prennent en ligne de compte les relations entre mémoires, identités et représentations sociales.

Mots-cléfs: identités; mémoire; représentations sociales

\section{ABSTRACT}

This article will discuss how socially constructed representations of the past concerning the role black people played in the memory of Brazil persist in the pupils' minds, requiring cautious approach by teachers. The discussion will be based on data collected from history classes of children 9 to in years old and will focus on existing links between memories, identities and social representations.

Keywords: memory; identities; social representations

\section{RESUMEN}

Este artículo analiza la manera en la que las representaciones construidas en el pasado sobre los negros en la historia de Brasil persisten en la imaginación de los alumnos, exigiendo una cuidadosa atención por parte de los profesores de historia para su deconstrucción y reconstrucción. El trabajo se basa en datos de un estudio llevado a cabo en las clases de historia con nińos de 9 a II años, y adopta como marco de interpretación las relaciones entre memorias, identidades y representaciones sociales.

Descriptores: memoria, identidades, representaciones sociales 


\section{Introduction}

$\mathrm{D}^{2}$ E NOMBREUX OBSTACLES CONCERNANT les nouveaux apprentissages en matière d'histoire et le développement de nouvelles identités sociales peuvent trouver leurs explications dans les représentations du passé, acquises socialement dans divers espaces et par l'intermédiaire de formes variées comme, entre autres, celle de la connaissance historique scolaire. Face à cela, deux défis sont à relever par les professeurs qui enseignent l'histoire. D'une part, celui de remettre en question les bases de la formation des mémoires historique et collective de l'identité de la nation, il s'agit ici de la nation brésilienne, en s'interrogeant sur les représentations sociales qui en sont tributaires. D'autre part, celui de l'élaboration de processus et de stratégies didactiques qui permettent aux sujets/apprenants de déconstuire et reconstruire des représentations constitutives de leurs identités sociales.

La thématique raciale a toujours été un élément central pour la compréhension de la société brésilienne et pour toute discussion tournant autour de la question d'identité nationale. Dans le contexte actuel de consolidation de la démocratie brésilienne et de mondialisation culturelle, l'éducation et surtout l'éducation historique est confrontée au défi de repenser ses finalités, ses contenus et ses formes d'acquisition de la connaissance. Dans un tel contexte, il est donc nécessaire de redéfinir les bases identitaires de la nation par la divulgation d'autres mémoires qui, jusqu'à maintenant, étaient peu présentes dans la mémoire historique et collective de la société brésilienne.

Dans le cadre de l'actuelle politique éducationnelle, on attribue à l'enseignement de l'histoire le rôle de former un nouveau citoyen qui serait capable de comprendre l'histoire de son pays et du monde à partir de mémoires multiples et originaires de la diversité des expériences humaines et non plus à partir de la vision, jusqu'alors prédominante, d'une mémoire univoque partant des élites ou d'un passé homogène.

La politique éducationnelle actuelle, par le biais de ces paramètres nationaux en matière de programmes scolaires- Parâmetros Curriculares Nacionais (PCNs) (Brasil, Secretaria de Educação Fundamental., 1996, 1998, 1998a) en brésilien - oriente donc les programmes, en particulier ceux d'histoire pour qu'ils aident à la construction d'identités socio-culturelles, démontrant ainsi une sensibililité aux dynamiques de la diversité des cultures nationales et l'intention de rompre avec une histoire autocentrée sur le national, l'Europe, la religion chrétienne et la culture blanche. L'altérité devient ainsi l'élément central de la formation des identités des nouvelles générations et des finalités de l'enseignement de l'histoire.

Cette perspective rejoint celle qui anime l'actuelle production historiographique et les nombreuses innovations relatives à l'enseignement de l'histoire au Brésil. Il est intéressant de signaler que les nouveaux programmes intègrent des données provenant des sciences de la pédagogie et surtout de la psychologie socio-cognitive qui considère le sujet/apprenant comme porteur d'expériences socioculturelles et actif dans son apprentissage. Une telle approche exige de redéfinir les méthodes et les processus d'enseignement.

Pour faire face à ces nouvelles finalités de l'enseignement de l'histoire et aux nouvelles perspectives des processus d'apprentissage, nombreuses sont les pratiques de salle de classe qui devront être remises en question. Un des défis les plus importants est, à 
nos yeux, celui de comprendre le va et vient toujours problématique qui existe entre l'acquisition de nouvelles connaissances et les représentations sociales que les élèves mobilisent au moment de leurs apprentissages.

Avant de nous pencher sur les résultats des recherches effectuées auprès d'enfants de 9 à II ans concernant leurs représentations des noirs dans l'histoire du Brésil et de tirer quelques conclusions utiles pour l'avancement de nos compréhensions du processus enseignement- apprentissage en histoire, nous présenterons notre compréhension conceptuelle des représentations sociales et de leur rôle dans les processus d'apprentissage et de formation des identités sociales, en nous renvoyant à les visions de la présence noire dans le processus de construction de la nation brésilienne.

\section{Le pays du mélange des races}

Le Brésil, plus que tout autre pays, est perçu comme une nation où le croisement des races est évident et se manifeste de forme harmonieuse. La spécificité de notre mélange de races, bien diffusé par la littérature, autant au Brésil qu'à l'étranger est cependant peu connu du point de vue de sa construction historique. Même s' il est vrai que la colonisation portugaise a favorisé ce mélange, le considérant comme un aspect stratégique de sa politique de peuplement, ce processus a fini par produire une formation sociale avec ses propres valeurs et particularités, chargées de contradictions. Il est nécessaire de rappeler ici que ce n'est qu'à partir des années vingt que les noirs - portant au centre du processus de croisement racial du Brésil colonial et impérial-commencent à intéresser l'historiographie brésilienne.

Les interprétations construites au fil du temps sur la place des noirs et de l'esclavage sont variées et souvent antagoniques. ${ }^{1}$ Grosso modo, nous pouvons dire que jusqu'aujourd'hui, on considère que la production historiographique concernant cette thématique a eu tendance à voiler l'existence des mélanges raciaux et de la production d'inégalités sociales et à méconnaître le rôle des noirs, indiens et métisses dans la formation de notre histoire.

Combattre les vestiges de l'esclavage, système qui a duré presque quatre siècles et dont la présence dans les relations et représentations sociales se manifestent non seulement dans l'exclusion des droits sociaux, mais aussi sous diverses formes de discrimination, préjugés et stéréotypes, est de mise aujourd'hui et fait l'objet de débats les plus variés au sein de la société brésilienne. Parmi d'autres questions, il est important de connaitre, du point de vue historique, les représentations sociales qui orientent les interprétations et les actions des élèves de toutes tranches d'âge afin qu'elles deviennent objet de problématisation et de reconstruction.

\section{Représentations sociales et formation des identités sociales}

D'un point de vue constructiviste, on considère que les connaissances préalables qui précèdent l'apprentissage scolaire jouent un rôle fondamental dans les processus d'apprentissage. Dans la relation sujet/objet, inhérente à tout acte cognitif, le cadre 
conceptuel du sujet est fondamental pour la sélection, l'organisation et la construction des informations avec lesquelles le sujet intéragit. Ainsi, ces idées dites préalables sont d'une grande importance pour déterminer ce qu'on apprend et comment on l'apprend.

En histoire et en sciences sociales en général, on entend par connaissances préalables un ensemble d'idées et de modes de penser ou de raisonner socialement construits. Il est donc important de rechercher quelles idées se font les élèves des divers concepts et thèmes de l'histoire, ce qu'est l'histoire pour eux, ce que représentent aussi pour eux les processus de production de la connaissance historique ainsi que les modes d'explication de l'histoire. Jusqu'à quel point ces connaisssances influencent-elles les nouveaux apprentissages et quelle est l'influence de l'enseignement dans la modification des connaissances préalables? Telles sont les questions principales posées par les chercheurs.

Nous argumentons en faveur de l'idée que la théorie des représentations sociales se montre d'une grande utilité pour les études des connaissances préalables que les élèves ont sur des thèmes relatifs à l'histoire. Pour Jodelet (1998), de qui nous avons pris ce concept de représentations, celles-ci sont «une forme de connaissance, socialement élaborée et partagée, ayant une vision pratique et participant à la construction d'une réalité commune à un ensemble social» (p. 36).

De ce point de vue, la connaissance acquise par le biais de processus sociaux n'est pas seulement le produit de caractéristiques inhérentes à l'homme; elle n'est pas non plus un simple réflexe des influences de l'environnement. La connaissance se présente comme une construction où intéragissent le sujet psychique et le monde extérieur. Les enfants et les adolescents ne sont donc pas contraints d'absorber et de refléter la connaissance du monde adulte comme un simple "miroir".

Entre l'acte d'internaliser les conceptions et celui de les exposer, il y a tout un processus où les objets sont réarticulés et où l'individualité de chacun se manifeste. En tant qu'agents, les sujets cherchent à trouver et à développer une identité, ils intéragissent de différentes manières avec le monde social, en incorporant et en recréant des objets et des symboles et en articulant les représentations sociales, la société et l'individu. Les représentations sociales, une fois constituées, réalisent un travail de médiation entre l'individu et la réalité sociale. A partir de là, le sujet se construit des représentations individuelles qui ne sont pas un simple reflet des représentations sociales, mais le fruit d'un processus de construction et de reconstruction de symboles socialisés et internalisés.

Comme le signale Jovchelovitch (2000)

L'émergence d'un moi en opposition au monde extérieur apparait en relation étroite (ou encore comme pré-condition) avec les transformations mentales qui permettent la représentation des choses et, de ce fait, le développement de la pensée symbolique et du langage. (p. 75)

Ainsi, les représentations se présentent comme la capacité de donner aux choses une "nouvelle forme» par le biais de l'activité psychique. Celle-ci implique une médiation entre le sujet et l'objet-monde. Comme le sujet s'insère dans une communauté con- 
crète (celle des groupes d'appartenance) et dans une communauté symbolique, il n'est pas condamné à simplement reproduire la réalité.

Il appartient à l'individu d'élaborer une tension permanente entre le monde, déjà constitué, et ses propres efforts pour devenir un sujet. Nous pouvons donc dire que le processus de construction de l'identité se développe à l'intérieur de la relation entre le sujet et le social, au travers des représentations sociales. En même temps que le sujet recrée la réalité sociale et ses représentations, il est aussi modifié dans sa propre relation avec le monde. De cette façon, les objets présents dans le milieu social apparaissent sous la forme de représentations, ils sont recréés par les sujets.

De plus, les représentations sociales pourraient de cette manière, être reliées autant à la circulation existant entre les divers groupes sociaux, passage d'un groupe social à l'autre, qu'aux transformations successives par lesquelles elles passent pendant les différents moments vécus par les élèves dans leur enfance et adolescence.

D’autres études viennent approfondir la compréhension théorique et méthodologique des représentations sociales. Jodelet (1998) attire notre attention sur la relation entre les représentations et le contexte de leurs constructions en signalant que la marque sociale des contenus et des processus de représentation se réfere aux conditions et aux contextes dans lesquels émergent les représentations, aux communications par lesquelles elles circulent et à leurs fonctions dans l'interaction avec le monde et avec les autres. Mary Spink (1993) insiste aussi sur l'importance des relations entre les représentations et les conditions de production, elle souligne que les représentations constituent une forme de connaissance qui ne pourra jamais être comprise à un niveau individuel. Cette connaissance ne peut être analysée qu' "en contrebalançant le contexte social dans lequel elle émerge, circule et se transforme» (p. 93). Jovchelovitch (2000) dit même que «les processus qui forment les représentations sociales sont partie intégrante de la communication et des pratiques sociales: dialogue, discours, rituels, modes de travail et de production, art, en bref, culture» (p. 79). Cependant, comme le signale Robert M. Farr (2000), « une représentation sociale vaut seulement la peine d'être étudiée si elle est relativement répandue dans la culture à laquelle on s'intéresse» (p. 46).

Finalement, il est bon de retenir la notion de Noyau Central des représentations sociales, défendue par d'autres chercheurs. Pour Abric et ses collaborateurs la structure des représentations sociales se présente de façon hiérarchique, organisée autour d'un noyau plus stable et résistant, le Noyau Central. Celui-ci, selon Abric (1994) est «constitué d'un ou de plusieurs éléments qui donnent son sens à la représentation au centre de ce noyau se trouvent les normes, les attitudes et les stéréotypes ${ }^{1}$. Comme nous le rappellera encore Moura (1996) «l'idée de noyau central se rapporte à celle de noyau figuratif, résultat de l'objectivation discutée par Moscovisci, cette dernière étant une structure imagé ayant, entre autres, des caractéristiques de prégnance, d'autonomie et de stabilité. Cette perspective permettra l'établissement de relations entre représentations sociales et mémoire collective. Comme l'exprime Abric (I994), le noyau central

est fortement marqué par la mémoire collective du groupe et par le système de norias auquel il se réfère. Il constitue de ce fait la base commune et collective- 
ment partagée des représentations sociales. C'est par lui que se définit l'homogénéité d'un groupe social. Il est stable, cohérent, résistant au changement, assurant ainsi une deuxième fonction, celle de la continuité et de la permanence de la représentation. Enfin, il est, d'une certaine manière, relativement indépendant du contexte social et matériel immédiat dans lequel la représentation est mise en évidence. (p. 78)

Par l'intermédiaire des outils conceptuels et méthodologiques de la théorie des représentations sociales que nous venons de voir, nous pouvons entreprendre des études qui rendent possible l'identification de représentations sociales qui, d'un côté, peuvent se constituer en ancrage-ce qui, pour Moscovisci, signifie «apporter aux catégories et aux images connues ce qui n'a pas encore été classifié et désigné ${ }^{2}-$ et qui de l'autre, peuvent se constituer en obstacles pour de nouveaux apprentissages. Dans l'étude qui suit, nous montrerons quelques représentations sociales qui structurent le monde collectif dans lequel les enfants se développent, aspect rarement exploré tant d'un point de vue épistémologique, telle la construction de la connaissance proposée par Piaget, que du point de vue de la psychologie cognitive sociale de Vygostky. Comme nous prévient Duven (2000), la théorie de Vygostky conçoit l'enfant comme un «sujet culturel »et dans cette conception, la culture est réduite à un ensemble de signes qui fonctionnent comme un instrument cognitif et exclut «ce sens où les signes, eux-aussi, expriment les valeurs de groupes sociaux particuliers» (p. I86).

Notre hypothèse est que, bien plus tôt qu'on ne l'imagine, les enfants forment dans leurs intéractions sociales quotidiennes des ensembles de représentations pour tisser des communications à l'intérieur de leur groupe et les aider à établir « un ordre, à s'orienter dans leur monde matériel et social et à le commander " (Duven, p. 268).

Ces représentations et modes de penser s'acquièrent par l'intermédiaire d'intéractions et d'expériences sociales qui surviennent dans les groupes auxquelles les enfants appartiennent, par l'intermédiaire d'autres véhicules de diffusion tels les médias, la télevision en particulier et, pourquoi pas, par l'intermédiaire de l'école. Il est donc certain que nos enfants connaissent déjà diverses formes de discrimination sociale et culturelle à l'égard des noirs, des indiens, des femmes, des pauvres ainsi que les notions de hiérarchie entre les différentes cultures. Très tôt, ils sont amenés à penser le changement social d'une manière linéaire et évolutive. Ces représentations et modes de penser expliquent les visions stéréotypées, simplistes et parfois fatalistes de la réalité.

\section{Représentations sociales des enfants concernant les noirs et l'esclavage au Brésil}

Dans le contexte de la recherche Construction de la connaissance et développement du raisonnement historique et de la citoyenneté que nous coordonnons, ${ }^{3}$ nous avons, tout au long de l'année académique de 200I, développé une thématique et cherché à remettre en question les visions consacrées par l'historiographie traditionnelle. Dans le cadre de cette étude, nous nous sommes proposés de vérifier les représentations sociales concernant «le mode de vie des noirs au Brésil» d'enfants de 9 -II ans scolarisés 
dans la quatrième année du cycle fondamental du Centre Pédagogique de l'UFMG. Nous avons demandé aux élèves qu'ils fassent des dessins sur les noirs et leur mode de vie et qu'ils écrivent, tout de suite après, des commentaires de leurs dessins.

Le développement du thème «le noir et l'esclavage au Brésil» s'est fait au travers de lecture de textes, d'interprétations de graphiques, de questionnaires et d'entretiens avec la famille. D’autres activités ont été développées en parallèle comme: les visites à l'exposition "Les 500 ans de Brésil» et au Musée de l'esclavage de Belo Vale Minas Gerais ou encore l'étude d'objets de la culture matérielle des esclaves; la présentation d'une « roda de capoeira» et la participation à un atelier de cuisine afro-brésilienne.

Le but de ces activités, basées sur des sources historiques variées, était de donner aux enfants la possibilité d'entrer en contact avec un passé, exprimé au travers de relations sociales, de pratiques et de traditions culturelles diverses et ses conséquences dans le présent.

Notre intention était de faire en sorte que les enfants perçoivent que le noir a résisté de différentes manières à sa condition d'esclave en conservant, par exemple, sa propre religiosité, ses danses et ses luttes, ses ornements corporels, ses coutumes, ses traditions culinaires, c'est-à-dire son identité culturelle. Par la simple observation du monde dans lequel il vit, l'enfant devrait être capable de noter, sans grande difficulté, la permanence de ces pratiques, faisant ainsi un exercice fondamental pour la compréhension et le développement du raisonnement historique.

Nous voulions aussi que l'imaginaire infantil et ses représentations concernant l'esclavage au Brésil ne restent pas uniquement lié à la condition de l'esclave en tant que victime dépourvue d'attitudes et de stratégies qui lui permettent de vivre un quotidien plus supportable et pluriel et que l'enfant comprenne que le travail de l'esclave ne se limitait pas à des travaux agricoles dans des régions rurales.

Cette perspective a cherché à se syntoniser avec, d'un côté, les interprétations historiographiques de caractère socioculturel, en insistant sur la singularité, la complexité et les contradictions de nombreuses relations entretenues dans le Brésil "esclavocrate" et de l'autre, avec l'enseignement rénové de l'histoire qui entend bien fournir des éléments pour la formation de nouvelles identités sociales à partir de nouvelles mémoires historiques. L'intention était donc d'identifier avec les enfants, les représentations sociales qui devraient se constituer en objet de déconstruction et d'ampliation pendant le processus d'enseignement/apprentissage de la thématique en question. Représentations qui pourraient correspondre aux vestiges laissés par l'esclavagisme dans la société brésilienne et qui se manifestent dans les relations sociales et la mémoire collective sous les formes les plus diverses de discrimination et d'exclusion sociale.

Nous passerons maintenant à la présentation et à l'analyse des données qui ont été recueillies (avant et après le développement de la thématique) sur les représentations sociales que les enfants ont des noirs dans l'histoire du Brésil.

\section{Représentation des noirs et de leur mode de vie}

Comme nous le verrons dans le tableau ci-dessous, les élèves, en représentant «les noirs et leur mode de vie» au travers de leurs dessins, ont présenté le travail esclave et le 
châtiment comme étant des situations normales dans la vie des noirs de l'époque. Sur 36 élèves, $56 \%$ ont représenté le travail esclave et 33\% le châtiment, environ $17 \%$ d'entre eux ont représenté le noir travaillant en étant châtié en même temps. On peut aussi noter une certaine variété dans les types de travaux représentés par les élèves. Certains abordent les travaux des champs (qui se résument à la plantation de café), d'autres représentent l'esclave cassant des cailloux, lavant du linge, portant des fardeaux, nettoyant la maison, "passant la serpillère", "construisant des choses”.

\begin{tabular}{|c|c|c|c|c|}
\hline & \multicolumn{3}{|c|}{ TYPES DE TRAVAIL } & \multirow{2}{*}{$\begin{array}{l}\text { RELATION } \\
\text { MAITRE/ESCLAVE }\end{array}$} \\
\hline & CHAMPS & DOMESTIQUE & AUTRES & \\
\hline Alessandra & & & $\begin{array}{l}\text { Casser des pierres/pous- } \\
\text { ser une brouette de terre } \\
\text { (minération) }\end{array}$ & Pieds enchaînés \\
\hline Aline C. & & Laver le linge & Porter des objets (broc) & \\
\hline Aline F. & & Passer la serpillère & & \\
\hline André & $\begin{array}{l}\text { Culture terra } \\
\text { (sarclage) }\end{array}$ & & & Pieds enchaînés \\
\hline Ariela & & & & Prison, fouet \\
\hline Bruno & & Balayer le sol & & Pieds enchaînés \\
\hline Carolina & Culture du café & & & \\
\hline Gabriela & & & Porter des fardeaux & $\begin{array}{l}\text { Pieds enchaînés fouet, } \\
\text { pilori }\end{array}$ \\
\hline Guilherme & & & Construire des habitations & Pieds enchaînés \\
\hline Gustavo & $\begin{array}{l}\text { Culture du café } \\
\text { (houe, tamis) }\end{array}$ & & & \\
\hline Henrique & & Balayer le sol & & Pieds enchaînés \\
\hline Isabella & & & Transporter des objets & \\
\hline Jeyson & & & Casser des pierres & Fouet \\
\hline João Vitor & & & & Fouet, mains enchaînées \\
\hline Júlia & & & Transporter des objets & $\begin{array}{l}\text { Prison et autres } \\
\text { Instruments }\end{array}$ \\
\hline \multicolumn{5}{|l|}{ Karolina } \\
\hline Laís & & & Transporter des objets & \\
\hline Luciano & & & Transporter du bois & Fouet \\
\hline Marcelo & & & Casser des pierres & Fouet, Pieds enchaînés \\
\hline Mateus & & & Casser des pierres & Fouet, Pieds enchaînés \\
\hline Natália & & $\begin{array}{l}\text { Ranger la cuisine } \\
\text { La chambre }\end{array}$ & & \\
\hline Nathan & & Laver le linge & Casser des pierres & $\begin{array}{l}\text { Corde au cou } \\
\text { Pieds enchaînés }\end{array}$ \\
\hline Octávio & & & Traire des vaches & \\
\hline Priscila F. & & & Transporter des objets & \\
\hline Priscila N. & & & Transporter des objets (bois) & \\
\hline Rodrigo & & & & $\begin{array}{l}\text { Esclave prisonnier et } \\
\text { Enchaîné }\end{array}$ \\
\hline Sarah & Culture du café & & & \\
\hline Suelen & & & Construction d'habitation & \\
\hline Vitor & Non spécifique & & & \\
\hline Sans nom & & & & Fouet \\
\hline TOTAL & 4 & 6 & 16 & 16 \\
\hline
\end{tabular}

Tableau I. Représentation des noirs et leur mode de vie (avant le développement de la thématique) 
Comme on peut l'observer, la majorité des enfants a associé le noir à l'esclavage et celui-ci à un travail manuel, extrèmement dur. Les esclaves en train de «casser des pierres» représentent l'extraction minière d'or ou de fer ou, plus symboliquement, le dur labeur: "J'ai dessiné les noirs en train de travailler, de casser des cailloux et de pousser une brouette pleine de terre" (Alessandra Xavier).

La société esclavagiste est toujours représentée comme une société basée sur un travail continu, sans repos: "l'esclave se repose seulement quand le maître dort» ou encore, "j’ai dessiné un château avec six esclaves qui travaillent et cinq portugais qui les surveillent» (Priscila F. Ferreira), "dans ce travail j'ai fait un portugais qui maltraite un noir» (Luciano Medrado). Ces commentaires montrent bien que pour eux le pouvoir de cette société était détenu par l'homme blanc-le portugais/colonisateur, propriétaire de la terre et de l'esclave. Les élèves n'imaginent pas qu'il puisse exister des lois régissant les rapports entre le maître et l'esclave. Tout se situe dans le domaine du privé.

Dans la dernière colonne du tableau I apparaissent les instruments de torture présents dans la majorité des "scènes" dessinées. Sur 36 élèves, un tiers représente le châtiment corporel comme faisant partie intégrante du quotidien de l'esclave, $80 \%$ d'entre eux représentent le noir-esclave dans une situation de travail et/ou de châtiment: «j'ai dessiné le noir en train de travailler et d'être châtié» (Matheus. F). La violence physique dépend du jugement du maître/propriétaire/blanc/portugais sur l'esclave/noir/captif. Le maître/blanc, représenté par la figure du colonisateur portugais surveille l'esclave, ou le régisseur, sur l'ordre du maître, bat, châtie, exerce un certain type de violence physique sur ceux qui désobeïssent aux ordres.

Les instruments de répression et de châtiment, surtout les chaînes et le fouet, occupent une place marquante. Les expressions faciales des noirs représentés sur les dessins expriment toujours la souffrance (pleurs, sueur, blessures) de ceux qui sont victimes et exclus de toute autre forme de vie. Ceci est confirmé par les commentaires écrits: «ils souffrent, travaillent, dépensent leur énergie, sont battus et portent des fardeaux» (Gabriela); "les nègres esclaves sont en train d'être fouettés et maltraités» (Marcelo).

Etre esclave signifie absence de liberté, soumission totale aux ordres du maître «ils vont laver le linge, ils sont enchaînés et ils cassent des cailloux, le boulet au pied.... on les pend» (Nathan). Parmi tous les élèves, seulement un a mentionné une forme de résistance: la tentative de détruire l'instrument de son châtiment "je vais briser mes chaînes " (André $\mathrm{M}$ ), exprimant par là la représentation d'une intention de fuite.

Il semble que pour ces enfants, le travail forcé et l'utilisation du châtiment ne représentent pas des mesures exemplaires mais, au contraire, synthétisent ce qu'a été la vie des noirs au Brésil. Nombreux enfants, dans leurs dessins, ont donné une place centrale aux scènes de châtiment. Au centre du dessin le noir est enchaîné à un poteau en train d'être frappé par le blanc/contremaître.

Ces images évoquent le célèbre tableau de Debret datant de I835, intitulé "Exécution des coups de fouet”. Sa reproduction peut être vue dans les livres didactiques, dans les films, les feuilletons télévisés, les expositions de musée. Elle est devenue ce queune image canonique, en ce qui concerne l'esclavage.

Ces scènes représentées peuvent donc être considérées comme une vision stéréotypée du noir et de sa condition d'esclave, entendons par stéréotype ce que Sperber (1985) 
appelle «une représentation collective dont il existe de nombreuses copies, des copies identiques de la représentation" (p. 196). En insistant sur la relation entre noir/esclave/châtiment, les enfants semblent généraliser le caractère exemplaire des châtiments qui touchent tous les noirs, pendant toute la durée de leur vie d'esclaves au Brésil.

Pour certains de ces enfants, la situation de domination du blanc sur le noir extrapole le "temps" de l'esclavage et se prolonge jusqu'aujourd'hui. Ce prolongement s'exprime sous la forme de relations autoritaires comme celle de la soumission du noir qui se résigne au dur travail manuel «j'ai dessiné un esclave qui porte le sac d'un homme» (Isabela R.); "les noirs travaillent, ils portent des caisses»; "une esclave passe la serpillère» (Aline F); "il va vite chercher du lait de vache» (Otávio S.).

Il est important de souligner l'absence d'images et de discours qui expriment la vie culturelle et religieuse des noirs et/ou les moments de bonne entente entre les maîtres et les esclaves. Etre noir, c'est être esclave et être esclave, c'est vivre dans une situation d'oppression permanente et de châtiment. Le mode de vie du noir se résume donc à la relation entre maître et esclave dans laquelle le travail et la violence sont toujours présents.

En observant le tableau 2, on pourra remarquer que sur un total de 29 enfants, I3 ont inclus des instruments de torture dans leurs dessins. Si nous considérons qu'au début de l'activité I6 sur 30 avaient aussi présenté divers instruments de torture et des scènes de châtiment associés au travail et à la vie des noirs réduits en esclavage, nous pouvons dire qu'il n'y a pas eu d'altérations dans leurs représentations sociales. Ce qui nous amène à réfléchir sur le peu d'efficacité des stratégies didactiques et pédagogiques employées pour la desconstruction de ces représentations. Le tableau 3 nous permettra de mieux visualiser ce que nous venons de dire et de comparer les dessins des enfants avant et après l'étude de la thématique.

Un enfant a exprimé par un discours de sens commun la complexité des relations sociales actuelles qui résultent du passé colonial et esclavagiste, montrant ainsi que le sens commun peut être resignifié, c'est-à-dire qu'il n'est pas imperméable aux changements quand il rentre en contact avec de nouvelles formes de connaissances.

En décrivant et dessinant une situation de dialogue entre noir/pauvre et blanc/riche ou entre le patron et le travailleur noir, il a dit, en imitant la voix du patron, "certaines personnes emploient des noirs... d'autres jurent: ho noir feignant; d'autres ne jurent pas: bonjour, comment tu t'appelles? Je veux être ton ami; il y en a d'autres qui emploient les noirs mais qui ne les payent pas: je ne vais pas te donner un sou; ou je vais te payer aujourd'hui». En prenant la voix du noir/pauvre/employé, il dit: «tu me donnes du travail? Et ma paye, chef et ma paye?" Finalement, en utilisant sa propre voix: "voilà, c'est de cette manière que les noirs vivent au Brésil. Il y en a qui souffrent de préjugés raciaux, d'autres non» (Ariela S. Athayde). 


\begin{tabular}{|c|c|c|c|c|c|c|c|c|c|c|}
\hline \multirow[b]{2}{*}{ NOM } & \multirow[b]{2}{*}{ 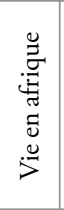 } & \multirow[b]{2}{*}{ 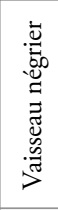 } & \multirow[b]{2}{*}{ 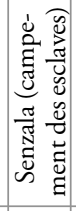 } & \multirow{2}{*}{$\begin{array}{l}\stackrel{\circ}{\text { है }} \\
\text { 름 }\end{array}$} & \multirow[b]{2}{*}{ 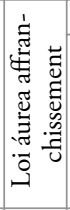 } & \multicolumn{3}{|c|}{ Types de travail } & \multirow{2}{*}{ 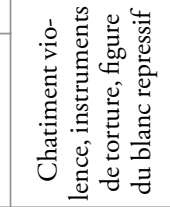 } & \multirow[b]{2}{*}{ 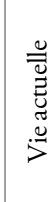 } \\
\hline & & & & & & 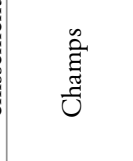 & 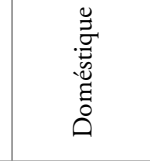 & 苞 & & \\
\hline Alessandra & Oui & & & & & $\begin{array}{l}\text { Moudre le } \\
\text { grain }\end{array}$ & & & Fouet, coutelas & \\
\hline Aline C. & & & & & & $\begin{array}{l}\text { Débrous- } \\
\text { saillement }\end{array}$ & & & Pilori, fouet & \\
\hline Aline F. & & Oui & & & & & & $\begin{array}{l}\text { Noire,vendeuse } \\
\text { de desserts }\end{array}$ & & \\
\hline Ana Paula & Oui & & & & & & & & & \\
\hline André & & & Oui & & Oui & & & & Fouet, pilori & \\
\hline Ariela & & & & & & & & & & Oui \\
\hline Bruno & & & & & & & & $\begin{array}{l}\text { Tissage Vente de } \\
\text { bijoux colifichets }\end{array}$ & & \\
\hline Bryan & & & & & & & & & Arme & \\
\hline Carolina & Oui & & & Oui & & Bétail & $\begin{array}{l}\text { Explication } \\
\text { seulement }\end{array}$ & «Minération » & Fouet & Oui \\
\hline Gabriela & Oui & Oui & & Oui & & & & & d & \\
\hline Gustavo & & & & & & $\begin{array}{l}\text { Explication } \\
\text { Seulement }\end{array}$ & $\begin{array}{l}\text { Explication } \\
\text { Seulement }\end{array}$ & & Poteau, fouet & \\
\hline Henrique & & Oui & & & & & & & & \\
\hline \multicolumn{11}{|l|}{ Isabella } \\
\hline Jeyson & & Oui & & & & & & $\begin{array}{l}\text { Cassage de cailloux, } \\
\text { Porteur d'eau }\end{array}$ & & \\
\hline João Vitor & & Oui & & Oui & & & $\begin{array}{l}\text { Lavage du } \\
\text { linge, luisine }\end{array}$ & & Poteau, fouet & \\
\hline Júlia & Oui & Oui & & & & & Cuisine & & Poteau, fouet & \\
\hline Karolina & & & & Oui & & & Cuisine & $\begin{array}{l}\text { Transporter des } \\
\text { objets }\end{array}$ & & \\
\hline Laís & & & & & & & $\begin{array}{l}\text { Lavage du } \\
\text { Linge }\end{array}$ & & & \\
\hline Luciano & & Oui & & & & Oui & & & Fouet & \\
\hline Mateus & Oui & & & & & & & & Fouet & \\
\hline Natália & & Oui & & & & Oui & & & Variété & \\
\hline Nathan & Oui & Oui & & Oui & Oui & $\begin{array}{l}\text { Débrous- } \\
\text { saillement }\end{array}$ & & & & \\
\hline \multicolumn{11}{|l|}{ Octávio } \\
\hline Priscila F. & Oui & & & & & $\begin{array}{l}\text { Cueillette } \\
\text { du café }\end{array}$ & & & & \\
\hline Priscila N. & & Oui & Oui & & & $\begin{array}{l}\text { Débrous- } \\
\text { saillement }\end{array}$ & $\begin{array}{l}\text { Lavage du } \\
\text { Linge }\end{array}$ & & & \\
\hline Rodrigo & & & & & Oui & Bétail & & & Variété & \\
\hline Sarah & Oui & & & & & & & & & \\
\hline Suelen & Oui & Oui & & Oui & & \multicolumn{3}{|c|}{ Ne donne pas d'explication } & $\begin{array}{l}\text { Explication } \\
\text { Seulement }\end{array}$ & \\
\hline Vitor & & & & Oui & & & & & & \\
\hline TOTAL & IO & 7 & 6 & 13 & 7 & II & 2 & Io & 3 & 2 \\
\hline
\end{tabular}

Tableau 2. Représentation des noirs et de leur mode de vie (après le développement de la thématique) 


\begin{tabular}{|c|c|c|}
\hline Nome & $\begin{array}{l}\text { Avant le développement } \\
\text { de la la thématique }\end{array}$ & Après le développement de la thématique \\
\hline Alessandra & Travail/châtiment & Travail/châtiment \\
\hline Aline C. & Travail & Travail/châtiment \\
\hline Aline F. & Travail & Travail/vaisseau négrier \\
\hline André & Travail/châtiment & châtiment/senzala/Loi Áurea \\
\hline Ariela & châtiment & Vie actuelle \\
\hline Bruno & Travail/châtiment & Travail \\
\hline Carolina & Travail & Travail/châtiment/quilombo/Loi Áurea/Afrique \\
\hline Gabriela & Travail châtiment & Afrique/vaisseau négrier/quilombo \\
\hline Gustavo & Travail & Travail/châtiment \\
\hline Henrique & Travail/châtiment & vaisseau négrier \\
\hline Isabella & Travail & Travail \\
\hline Jeyson & Travail/châtiment & Travail/vaisseau négrier \\
\hline João Vitor & châtiment & Travail/châtiment/vaisseau négrier/quilombo \\
\hline Júlia & Travail/châtiment & Travail/châtiment/Afrique/vaisseau négrier \\
\hline Karolina & & Travail/quilombo \\
\hline Laís & Travail & Travail \\
\hline Luciano & Travail/châtiment & Travail/châtiment/vaisseau négrier \\
\hline Mateus & Travail/châtiment & châtiment/Afrique \\
\hline Natália & Travail & Travail/vaisseau négrier \\
\hline Nathan & Travail/châtiment & $\begin{array}{l}\text { Travail/châtiment/Afrique/vaisseau négrier/quilom- } \\
\text { bo/Loi Áurea }\end{array}$ \\
\hline Octávio & Travail & \\
\hline Priscila F. & Travail & Travail/Afrique \\
\hline Priscila N. & Travail & Travail/vaisseau négrier/senzala \\
\hline Rodrigo & châtiment/Travail & Travail/châtiment/Loi Áurea \\
\hline Sarah & Travail & Loi Áurea \\
\hline Suelen & Travail & Travail/châtiment/quilombo/vaisseau négrier/Afrique \\
\hline Vitor & & Quilombo \\
\hline
\end{tabular}

Tableau 3- changement et permanences de representations

D'après la seconde colonne du tableau 3, il est évident que bien que 7 enfants aient continué à associer travail et châtiment, 7 autres ont entamé leur processus de déconstruction de ce qui est en train de se configurer comme étant le noyau central de la représentation actuelle du noir dans la formation de l'histoire du Brésil. Desconstruire la représentation de l'univers social du noir victime et chosifié ou sa négation en tant que sujet dans la relation maître/esclave, n'est pas une tâche facile pour les professeurs d'histoire.

Létude de quelques commentaires des deux groupes d'enfants nous aidera à mieux analyser ce que nous venons de faire. Parmi ceux qui ont maintenu leurs représentations sociales, certains ont dit "les blancs tuent les noirs parce qu'ils sont racistes» (Braian C. Silva); "les esclaves travaillent et reçoivent des châtiments» (Aline C. Rocha); les noirs travaillent et sont châtiés au Brésil» (Alessandra G. Xavier); "ils se reposent seulement quand les maîtres dorment" (André A. Santos).

On peut aussi verifier par le tableau 3 que 7 enfants ont déconstruit la relation travail/violence/châtiment comme "noyau central" de la vie des noirs au Brésil; ce qui peut être vu comme leur compréhension de ce qu'a été l'esclavage au Brésil. Ces élèves considèrent la résistance des esclaves comme l'élément qui s'infiltrait dans les relations maître/esclave au Brésil. Le châtiment était la punition pour la résistance qui se mani- 
festait, en général, par la fuite: "mon dessin montre la vie des noirs: le travail, les fuites, les châtiments, les échanges et les lettres d'affranchissement qui leur permettaient d'être libérés" (Rodrigo Viegas A. Guerra).

La forme par laquelle cet enfant a structuré son discours et disposé les éléments de son dessin nous laisse penser que, dans sa nouvelle compréhension des relations entre le maître et l'esclave, le châtiment était la conséquence de la tentative de fuite, traduisant ainsi la forme de résistance à l'esclavage la plus utilisée par les esclaves.

Dans son discours ce même enfant emploie le mot "échanges" montrant ainsi qu'il a assimilé qu'entre blancs, noirs et indiens il y a eu des relations interculturelles non marquées par la violence, surtout de la part des premiers (les blancs) contre les deux autres peuples. D'autres enfants ont inclus dans leurs dessins et commentaires la présence des quilombos, les lieux où se réfugiaient les nègres marrons qui tentaient d'échapper ainsi à leur recapture par les chasseurs d'esclaves, les capitaines “do mato", des fourrés.

Voyons comment ils ont exprimé leur nouvelles connaissances: "quand ils fuyaient, ils allaient dans les quilombos et se cachaient des capitaines "do mato". Les quilombos étaient toujours pleins" (Gabrielle M. Silva); "beaucoup de noirs s'enfuyaient chez les indigènes et dans les quilombos, c'était leur manière à eux de résister" (Carolina Afonso Carvalho.) Un enfant a parlé de l'orgueil et de la dignité morale des noirs comme étant une manifestation de la résistance à leur condition d'esclave: "les esclaves ont été maltraités, ont travaillé mais n’ont pas perdu le respect» (João Victor L. Araújo).

\title{
Considérations finales
}

\author{
Juntaremos tantos grilhóes \\ Quanto for possível \\ E mais quatrocentas misérias \\ Então trocaremos tudo por flores \\ Para enfeitar o enterro \\ Dessa coisa estranha: racismo \\ (Canção para um negro abandonado de Ele Semog) ${ }^{4}$
}

Les résultas que nous venons de présenter s'encadre, comme nous l'avons déjà dit, dans le contexte des recherches sur la Construction de la connaissance et développement du raisonnement historique et de la citoyenneté, que nous avons coordonnées et développées durant l'année 200I.

Par le biais de cette thématique relative aux noirs dans l'histoire du Brésil, nous avons cherché à: a) détruire les représentations sociales dont les enfants sont porteurs - nombreuses d'entre elles se basant sur des relations de discrimination raciale et sociale des noirs au Brésil et justifiant ces dernières; b) permettre aux enfants de construire de nouvelles connaissances historiques basées sur une vision plus complexe, plurielle et contradictoire concernant le role des noirs dans la formation de notre histoire. Par ces 
deux opérations complémentaires, nous avons eu l'intention de contribuer à la construction de nouvelles références identitaires.

Ce que cette étude nous a permis de discuter et d'affirmer doit être considéré comme étant une première approche théorique et méthodologique du traitement des représentations sociales que les enfants se font à l'égard de la présence des noirs dans l'histoire du Brésil et de leurs destructions et reconstructions. Les données que nous avons présentées et leurs interprétations sont loin d'épuiser les possibilités qu'ouvrent ce domaine.

Cependant, bien que limitée à un cas, cette étude nous a permis, entre autres, d'obtenir des indices sur la façon dont des représentations sociales, inicialement partagées par un même groupe-comme l'était celle de l'esclave victime et non sujet-n'ont pas été déconstruites par tous et que pendant cette déconstruction, tous n'ont pas attribué le même sens à cet objet. Le fait de ne pas avoir été déconstruit par tous nous invite, d'une part, à remettre en question l'efficacité des stratégies pédagogiques et, d'autre part à se rendre compte que des représentations sociales très cristallisées peuvent fonctionner comme obstacles pour de nouveaux apprentissages et constituent un défi à long terme.

Cette étude nous a aussi permis de voir que la construction de nouvelles connaissances sous d'autres conditions et perspectives, mobilise d'autres représentations sociales qui existent déjà. Nous nous référons ici à la représentation, socialement partagée, de l'individualisation des actes de nature historique. Il nous est apparu que plusieurs étudiants, qui avaient pourtant compris l'existence de forces et d'intérêts en conflit dans la société brésilienne, ont attribué l'abolition de l'esclavage à un acte généreux de la Princesse Isabel, héroine nationale qui fait partie de la mémoire collective de la nation. L'histoire et les individus semblent avoir de grandes difficultés à abandonner leurs mythes! La représentation sociale de l'abolition mériterait, en effet, d'être étudiée plus sérieusement.

Nous pouvons donc dire que cette étude nous a apporté des indices sur l'importance qu'ont les mythes et l'héritage culturel sur les représentations sociales qui, de ce fait, sont résistantes et moins susceptibles de changements rapides. Cette réflexion ne fait que renforcer les études de Guareschi et Jovchelovitch et Farr (p. 20). Ainsi, si nous désirons contribuer aujourd'hui, par l'enseignement de l'histoire, à la formation de nouvelles mémoires, identités et relations sociales nous ne pouvons pas ignorer les représentations sociales que nos élèves portent en eux. Ce défi devra être relevé le plus rapidement possible.

Cette étude nous a aussi permis de confirmer que les élèves non seulement discutent de la thématique dans leurs groupes d'appartenance mais qu'ils ont aussi certainement vécu des expériences de discrimination raciale envers les noirs ou même envers d'autres cultures. On peut aussi supposer qu'ils ont établi des contacts avec les productions télévisées ou le cinéma par l'aussi lesquels circulent des représentations relatives à cette thématique. L'univers esclave a toujours été la prédilection des auteurs et producteurs des feuilletons historiques.

Il est donc nécessaire d'étudier les différents réseaux de formation des représentations sociales sans pour autant oublier que celles-ci conservent également des relations 
avec les connaissances scolaires, avec les manuels didactiques auxquels les enfants et leurs familles ont et auront accès dans leurs trajectoires scolaires. ${ }^{5}$

En plus de cela, nous devons pour mieux comprendre les processus de formation des représentations sociales, ajouter à ces études la dimension ethnique, celle du genre, de l'appartenance religieuse, des niveaux de scolarité et des différents contextes socioéconomiques. Comme nous le rappelle Robert M. Farr (2000) «les représentations sont autant présentes dans "le monde" que dans "l'esprit" et elles doivent être étudiées dans les deux contextes" (p. I32).

Pour conclure, nous dirons que le domaine des représentations sociales est un domaine de luttes sociales discursives et de manipulation comme celui de la mémoire collective et c'est pour cela que nous devons, comme le dit Le Goff (I988), "faire en sorte que la mémoire collective serve à la liberation et non à l'asservissement des hommes» (p. 177).

\section{Notes}

I Nous avons lu dans Moura MariaLúcia Seidl Práticas sócio-culturais, construção compartilhada de conhecimento e representaçôes sociais. In Trindade, Zeidi Araújo e Camino Cleonice. Cognição social e Juízo moral. Recueil de l'ANPEPP, vol.ı n:6, Sept. 1996, p. 32 et 33. Pour plus de details, lire Abric, J.C: Pratiques sociales et representations, Paris: Presse Universitaire de France, 1994.

2 Farr, Robert, \& Moscovici, Serge (Eds.) Social representations, Cambridge: Cambridge University Press, 1984, p. 30. Cité par Guarschi Pedrinho, A. Sem dinheiro não há salvação ancorando o bem e o mal entre pentecostais. In Guareschi Pedrinho e Sandra Jovchelovitch (orgs), op. cit. p. 201.

3 Lesclavage et le rôle des noirs dans la formation du processus historique brésilien ont été le thèmes de diverses interprétations d'historiens contemporains. Parmi eux nous soulevons les études de Kátia Matoso, Ser escravo no Brasil, São Paulo, Brasiliense, 1982; Jacob Gorender A escravidão reabilitada, São Paulo Ática, I990; Fernando Henrique Cardoso, Capitalismo e escravidão no Brasil meridional, São Paulo: Difusão Européia do Livro, 1962; Leila Mezan Algranti, O feitor ausente, Petrópolis, Vozes, 1988. Les interprétations qui sont à la base des représentations sociales seront appronfondies au moment de la discussion des données concernant les recherches présentées dans ce travail.

4 Nous rassemblerons autant de chaînes que possible

Et quatre cents misères en plus

Nous échangerons le tout contre des fleurs

Pour décorer l'enterrement

De cette étrange chose qu'on appelle racisme

(chanson pour un noir abandonné de Ele Semog)

5 Les manuels didactiques sont porteurs et diffuseurs de la mémoire de la nation et, avec d'autres productions culturelles, sont en train de se modifier. Au Brésil, on rencontre une rupture avec l'histoire traditionnelle et conservatrice qui exprime le point de vue des élites, des vainqueurs et des dominateurs. L'opposition a commencé dans les années 70 et s'est affirmée dans les années 70 et 80 . Elle se basait surtout sur l'histoire marxiste, tendance staliniste et orthodoxe. A partir de la fin 80 , la nouvelle histoire culturelle française et le révisionnisme marxiste anglais ont provoqué chez les historiens contemporains une contestation de l'interprétation marxiste orthodoxe et des modèles réductionnistes et généralisateurs qui étaient de mise pour l'analyse de l'expérience historique de l'esclavage au Brésil. 


\section{Références}

Abric, J.C. (1994). Pratiques sociales et representations. Paris: Presses Universitaires de France. Farr, Robert, \& Moscovici, Serge (Eds.) (1984). Social representations. Cambridge: Cambridge University Press.

Algranti, L.M. (1988) O feitor ausente, Petrópolis, Vozes.

Brasil. Secretaria de Educação Fundamental (1996). Parâmetros Curriculares Nacionais. História: ensino de primeira à quarta série. Brasília: MEC/sEF.

Brasil. Secretaria de Educação Fundamental (1998). Parâmetros Curriculares Nacionais. História: ensino de primeira à quarta série. Brasília: $\mathrm{MEC} / \mathrm{sEF}$.

Brasil. Secretaria de Educação Fundamental (1998a). Parâmetros Curriculares Nacionais. História: ensino de quinta à oitava série. Brasília: MEC/sEF.

Cardoso, F.H. (1962). Capitalismo e escravidão no Brasil meridional. São Paulo: DifusãEuropéia do Livro.

Gorender, J. (1990). A escravidão reabilitada, São Paulo Ática.

Duven, G. (2000). Les enfants en tant qu'acteurs sociaux: représentations sociales en développement. Textos em representaçōes sociais. Petrópolis: Vozes Editora.

Guareschi, P.A. (2000). Sem dinheiro não há salvação ancorando o bem e o mal entre pentecostais. Dans Guareschi, P. \& Jovchelovitch, S. (Orgs.), Textos em representaçóes sociais. Petrópolis: Vozes Editora.

Jodelet, D. (1998). Répresentations sociales: phénomènes, concept et théorie. Dans Moscovici, S. (Ed.). Phychologie sociale (2 ed.). Paris: Presses Universitaires de France.

Jovchelovitch, S., Guareschi, P., \& Farr, R. (Orgs) (2000). Textos em representaçöes sociais. Petrópolis: Vozes Editora.

Jovchlovitch, S. (2000). Vivendo a vida com os outros: intersubjetividade, espaço público e representaçóes sociais. Dans Guareschi, P., \& Jovchelovitch, S. (orgs). Textos em representaçôes sociais. Petrópolis: Vozes Editora.

Le Goff, J. (1988). Histoire et Mémoire. Paris: Gallimard.

Matoso, K. (1982). Ser escravo no Brasil. São Paulo: Brasiliense.

Seid, M.M. (1996, Septembre). Práticas sócio-culturais, construçáo compartilhada de conhecimento e representaçóes sociais. Dans Trindade, Zeidi Araújo e Camino Cleonice. Cognição social e Juízo moral. Recueil de L'anpepp, I(6) 32-33.

Spink, M.J.P. (1993). O estudo empírico das representaçôes sociais. Dans Spink, M.J.P. $O$ conhecimento no cotidiano: as representaçōes sociais na perspectiva da psicologia social. São Paulo: Brasiliense. 